\title{
60. \\ Determinación de un índice de riesgo y vulnerabilidad en poblaciones costeras: estudio de caso Portobelo, provincia de Colón
}

\section{Determination of a Risk and Vulnerability Index in Coastal Populations: Case Study Portobelo, Province of Colón}

\author{
Ariel Antonio Grey G. ${ }^{\text {I* }}$, Alides Canto ${ }^{1}$, Yarisel González ${ }^{l}$, Kelsyn Barria ${ }^{l}$, Itzel Castillo ${ }^{l}$ \\ ${ }^{1}$ Facultad de Ingeniería Civil, Universidad Tecnológica de Panamá, Panamá \\ *Autor de correspondencia: ariel.grey@utp.ac.pa
}

RESUMEN-El impacto ocasionado por las actividades humanas convierte a las zonas costeras en áreas vulnerables. Es por esto por lo que surge la necesidad de desarrollar un índice de riesgo y vulnerabilidad. El objetivo de la investigación es lograr determinar un índice de riesgo y vulnerabilidad a partir de la calidad fisicoquímico y microbiológico de las aguas superficiales marino-costeras y factores socioeconómicos utilizando como caso de estudio la ciudad costera de Portobelo, provincia de Colon. La metodología se dividió en 3 partes: determinación de un índice de calidad de agua marina costero, el análisis de amenazas y vulnerabilidades y la presentación del índice de riesgo y vulnerabilidad. Como resultado se obtuvo que el Índice de calidad de agua marina fue representada por 71.71, una calidad buena a regular. Se determino las vulnerabilidades, física con un valor de 2.0, moderado a bajo; educativas de 2.7, bajo a moderado; socioeconómicas de 3.6, moderado a alto; y ambientales de 3.7, moderada a alta. Se obtuvo un índice de Riesgo y Vulnerabilidad de 3.0, considerado moderado. El índice de Riesgo y Vulnerabilidad representa una herramienta que puede ser considerada para determinar un valor que defina el grado de riesgo, amenaza y vulnerabilidad, que está expuesto una comunidad costera y poder implementar medidas de mitigación y concientización a la población para evitar que las amenazas sean de un impacto mayor, provocando desastres mayores y evitando la contaminación de los recursos.

Palabras clave-Índice de Riesgo y Vulnerabilidad, Factores Socioeconómicos, Índice de Calidad del Agua.

ABSTRACT - The impact caused by human activities makes coastal areas vulnerable areas. This is why the need arises to develop a risk and vulnerability index. The objective of the research is to determine a risk and vulnerability index based on the physicochemical and microbiological quality of the marine-coastal surface waters and socio-economic factors using the coastal city of Portobelo, province of Colon as a case study. The methodology was divided into 3 parts: determination of a coastal marine water quality index, the analysis of threats and vulnerabilities and the presentation of the risk and vulnerability index. As a result, it was obtained that the Marine Water Quality Index was represented by 71.71, a good quality to regulate. Vulnerabilities were determined, physical with a value of 2.0, moderate to low; 2.7, low to moderate educational; socioeconomic from 3.6, moderate to high; and environmental of 3.7, moderate to high. A Risk and Vulnerability index of 3.0 was obtained, considered moderate. The Risk and Vulnerability Index represents a tool that can be considered to determine a value that defines the degree of risk, threat and vulnerability that a coastal community is exposed to and can implement mitigation and awareness measures to the population to prevent threats are of a greater impact, causing major disasters and avoiding the contamination of resources.

Keywords-Risk and Vulnerability Index, Socioeconomic Factors, Water Quality Index.

\section{Introducción}

En las zonas costeras se ha evidenciado la latente amenaza por contaminantes de diferentes fuentes terrestres, que llegan a ella no sólo a través de fuentes naturales como ríos y quebradas sino a través de las conexiones de tuberías urbanas que depositan allí sus aguas residuales [1].

El impacto ocasionado por las actividades humanas convierte a las zonas costeras en áreas vulnerables para la población. Es por esto, por lo que surge la necesidad

Citación: A. Grey, A. Canto, Y. González, K. Barría y I. Castillo, "Determinación de un índice de riesgo y vulnerabilidad en poblaciones costeras: estudio de caso Portobelo, provincia de Colón", Revista de I+D Tecnológico, vol. 16, no. 2, pp. (no_modificar), 2020.

Tipo de artículo: Original. Recibido: 12 de septiembre de 2019. Recibido con correcciones: 20 de septiembre de 2019 Aceptado: 7 julio de 2020.

DOI.

Copyright: 2020 A. Grey, A. Canto, Y. González, K. Barría y I. Castillo. This is an open access article under the CC BY-NC-SA 4.0 license (https://creativecommons.org/licenses/by-nc-sa/4.0/). 
de desarrollar un índice de riesgo y vulnerabilidad de las zonas costeras ante la contaminación marina, debido a que estas áreas exigen un manejo adecuado que asegure su conservación, desarrollo sostenible y preservación de los valores culturales de las comunidades tradicionalmente allí asentadas [2].

En Panamá, la contaminación de las aguas marinas puede ser observada con facilidad y con mayor énfasis en la vertiente pacífica, ya que es justo ahí donde se concentra la mayor cantidad de zonas pobladas o urbanas del país, en contraste con la vertiente Atlántica, donde existen pocas áreas pobladas, centrándose ésta en el área costera de Colón y Bocas del Toro [3].

La confluencia de las actividades humanas, sumada la falta de planificación y control y la inadecuada disposición de los residuos sólidos y domésticos, vertimientos provenientes de fuentes puntuales y difusas que llegan a través escorrentías fluviales o urbanas [4-6], convierten a las zonas costeras en áreas frágiles y limitadas porque pueden cambiar las características de la calidad del agua, con efectos potenciales sobre las población humana y los ecosistemas marino-costeros [5] incrementando la vulnerabilidad y disminuyendo la capacidad de adaptación [4-7].

Las costas panameñas se han transformado a lo largo de los años gracias al aumento del turismo, el aumento de la población no planificado y la afectación a los recursos naturales, a esto se adjudica el ascenso del nivel del mar debido al caliento global con el deshielo de los cascos polares que pueden incrementar significativamente el nivel mundial y con ello afectar costas de todo mundo incluyendo las nuestras de manera significativa.

Teniendo en cuenta lo antes expuesto, surge la necesidad de desarrollar un índice de riesgo y vulnerabilidad de las zonas costeras ante la contaminación marina, debido a que estas áreas exigen un manejo adecuado que asegure su conservación, desarrollo sostenible y preservación de los valores culturales de las comunidades tradicionalmente allí asentadas [2].

El objetivo es lograr determinar un índice de riesgo y vulnerabilidad a partir de la calidad de las aguas superficiales marino-costeras y factores socioeconómicos aplicado a zonas costeras utilizando el área de estudio, la ciudad de Portobelo, provincia de Colón. Se analizó el riesgo y la vulnerabilidad usando la amenaza por contaminación fisicoquímica, inundaciones y deslizamientos de tierra en la zona costera de la ciudad de Portobelo. También, se evaluó aspectos socioeconómicos, aspectos físicos, educativos que permitan explorar los efectos producidos por el deterioro ambiental en los habitantes del área de estudio.

\section{Materiales y Métodos}

La metodología empleada en el proyecto se dividió en 3 fases: la determinación de un Índice de Calidad de Agua, el análisis de las amenazas y vulnerabilidades de la comunidad y por último la determinación de un índice de riesgo y vulnerabilidad para la zona costera de la ciudad de Portobelo.

La calidad de agua se mide de acuerdo con distintos parámetros (físicos, químicos y microbiológicos) mediante los cuales se cuantifica el grado de alteración de las cualidades naturales y se le clasifica para un uso determinado como lo es el consumo, la agricultura, pesca, natación o agua que se utiliza en la industria [8].

Los indicadores ICA básicamente son una expresión de un número de parámetros que permiten valorar el recurso hídrico para un determinado uso [9]. Se aplicará el método de Promedio Aritmético Ponderado (ecuación \#1), en donde se tomará en cuenta los pesos relaticos asignados a cada variable, que son: $\mathrm{pH} 0.12$; Fosfato $0.10 ; \quad$ Nitrato $0.10 ;$ OD 0.17 ; Turbiedad 0.08 ; Temperatura 0.10; $\mathrm{DBO}_{5}$ 0.10; Coliformes Totales 0.11 [10].

$$
I C A=\sum_{i=1}^{9} \operatorname{Subi} \times W i
$$

En la ecuación (1), Sub $\mathrm{b}_{\mathrm{i}}$ es el subíndice del parámetro y Wi es el peso relativo.

Según Guillén et al., el ICA indica el grado de contaminación del agua a la fecha del muestreo y esta expresado como porcentaje del agua pura; así, agua altamente contaminada tendrá un valor cercano o igual a cero (0), en tanto que el agua en excelentes condiciones tendrá un valor de este índice cercano al cien (100) [8], [11].

Tabla 1. Escala de Valores del ICA

\begin{tabular}{|c|c|}
\hline Categoría & Rango numérico \\
\hline Pésima & $0-25$ \\
\hline Mala & $26-50$ \\
\hline Regular & $51-70$ \\
\hline Buena & $71-90$ \\
\hline
\end{tabular}


Fuente: [12].

\begin{tabular}{l|r} 
Excelente & $91-100$
\end{tabular}

La metodología para el análisis de amenazas y vulnerabilidad que fue empleada se dividió en dos fases, la primera consiste en poder recopilar toda la información relevante de la zona de estudio por medio de revisión bibliográfica y a través de tecina de encuestas.

La segunda fase se identificó y evaluó de las amenazas y vulnerabilidades presentes en la comunidad. Para ello se elaboró una metodología llamada "matriz para el análisis de amenazas y vulnerabilidades", la cual tiene como objetivo evaluar de manera rápida las vulnerabilidades antes las amenazas que se presentan en la comunidad.

Las amenazas evaluadas serán de tipo socio-naturales (inundaciones o deslizamientos de tierras) y de tipo antropogénicas (Contaminación del agua).

Para evaluar las amenazas de tipo socio natural se aplicaron encuestas realizadas a la comunidad, para obtener el porcentaje de viviendas que fueron afectadas por alguno de estos fenómenos. Para un porcentaje mayor de $90 \%$ de la población afectada por estos fenómenos el grado de amenaza muy alto con un puntaje de 5 y para un porcentaje menor de $10 \%$ el grado de amenaza será muy bajo con un puntaje de 1 .

Para las amenazas de tipo antropogénicas se analizó el ICA obtenido, para ello se aplicará la siguiente ponderación:

Tabla 2. Grado de Amenaza para el Índice de Calidad de Agua

\begin{tabular}{|c|c|c|}
\hline $\begin{array}{c}\text { Rango numérico } \\
\text { en el ICA }\end{array}$ & $\begin{array}{c}\text { Grado de } \\
\text { amenaza }\end{array}$ & Puntaje \\
\hline $0-25$ & Muy alto & 5 \\
\hline $26-50$ & Alto & 4 \\
\hline $51-70$ & Moderado & 3 \\
\hline $71-90$ & Bajo & 2 \\
\hline $91-100$ & Muy bajo & 1 \\
\hline
\end{tabular}

El Índice de amenaza total se calcula el valor promedio de las diferentes amenazas analizadas, aplicando la siguiente ecuación:

$$
I A=\frac{A A+A S N}{2}
$$

En la ecuación (2), IA es índice de Amenazas, AA Amenazas antropogénicas, ASN Amenazas Socio naturales.
Para la evaluación de los diferentes tipos de vulnerabilidades (físicas, socioeconómicas, educativas y ambientales) se determinó algunas variables e indicadores. Para ello se tomó en cuenta los resultados de las encuestas aplicada a la población. Dependiendo de las variables evaluadas se aplicó rangos de porcentaje para cada indicador y poder obtener un puntaje para determinar el grado de vulnerabilidad.

Para cada tipo de vulnerabilidad se consideró aquellos indicadores que permitan poder evaluar efectivamente la vulnerabilidad antes las distintas amenazas que enfrenta la comunidad.

Para las vulnerabilidades físicas tiene que ver con la ubicación física de los asentamientos y con la calidad de las condiciones de las viviendas [13], se evaluó el tipo de material utilizado en las viviendas y la cantidad de habitantes en la comunidad.

Vulnerabilidades socioeconómicas evalúan las condiciones de vida generales de una comunidad, se analizó los factores en donde se determina la población desempleada, el rango salarial, si la población cuenta con casa propia y si cuentan con servicios básicos como sistemas de acueducto, electricidad, internet, teléfono y radio.

Vulnerabilidades ambientales son aquéllos que se relacionan con la manera cómo una comunidad determinada "explota" los elementos de su entorno debilitándose a sí misma y debilitando a los ecosistemas [13]. Se evaluaron variables como: si la comunidad cuenta con sistema de alcantarillado y un tratamiento de agua residuales y la eliminación de los desechos sólidos que fácilmente pueden indicar un deterioro en el ecosistema afectando de manera indirecta a la población.

Vulnerabilidad educativa, bien se dice que si la población esta lo adecuadamente educado o que tenga conocimiento sobre los peligros potenciales que pueden enfrentar en sus comunidades y de poder prevenirlos se puede bajar de una manera significativa el grado de vulnerabilidad en la población, para ellos se evaluó el nivel de estudio de los pobladores, si tienen conocimiento sobre medidas preventivas ante amenazas por inundaciones y deslizamientos y también se estudiara el conocimiento sobre el cambio climático.

Para obtener el valor del índice de vulnerabilidad total se calcula el valor promedio de las diferentes vulnerabilidades analizadas, aplicando la siguiente ecuación: 


$$
I V=\frac{V S+V F+V A+V S}{4}
$$

En la ecuación (3), IV es índice de vulnerabilidad, VF vulnerabilidad física, VA vulnerabilidad Ambiental y VE Vulnerabilidad educativa.

En la matriz se busca evaluar e identificas aquellas variables e indicadores que están detrás de las amenazas $\mathrm{y}$ vulnerabilidades, en donde se clasificaran en vulnerabilidades o amenazas de tipo muy bajas hasta muy altas dándole un puntaje de 1 a 5 . Cada clase de vulnerabilidad o amenaza se refleja con un color de alerta.

Tabla 3. Grado de Amenazas y Vulnerabilidades
\begin{tabular}{|c|c|}
\hline Grado & Puntaje \\
\hline Muy Bajo & 1 \\
\hline Bajo & 2 \\
\hline Moderado & 3 \\
\hline Alto & 4 \\
\hline Muy alta & 5 \\
\hline
\end{tabular}

El Índice de riesgo y vulnerabilidad se obtendrá a partir de la evaluación de los factores socioeconómicos y el índice la calidad de agua marina en la zona de estudio ubicada en la zona costera de la ciudad de Portobelo, Provincia de Colon y por último se aplicó la ecuación (4) y para determinar el grado de riesgo se utilizó la tabla 4.

$$
I R V=\frac{a \times b}{2}
$$

En la ecuación (4), IRV es el índice de riesgo y vulnerabilidad, "a" es el valor de amenaza total y "b" es el valor de vulnerabilidad total.

Tabla 4. Ponderación para el grado de riesgo

\begin{tabular}{|c|c|}
\hline Ponderación & Grado de riesgo \\
\hline$<1.5$ & Muy Bajo \\
\hline $1.6-2.5$ & Bajo \\
\hline $2.6-3.5$ & Moderado \\
\hline $3.6-4.5$ & Alto \\
\hline$>4.6$ & Muy alta \\
\hline
\end{tabular}

\section{Resultados y discusión}

Se realizaron 5 muestreos en Portobelo desde julio 2017 hasta marzo 2019. Los resultados presentados se muestran por estaciones que corresponden al promedio de los 5 muestreos. Los parámetros que fueron evaluados son: Potencial de Hidrogeno ( $\mathrm{pH})$, Fosfato (PO4), Nitratos (NO3), Oxígeno Disuelto (O.D.), Demanda Bioquímica de Oxigeno (BDO5), Turbiedad (Turb.), Coliformes Totales (C.T), Temperatura (Temp.), Solidos Totales (S.T.).

Para la evaluar el índice se determinó el valor del subíndice del ICA para cada parámetro por estación, en la tabla 5, se resumen los resultados obtenidos. Con respecto a los sólidos disueltos totales se asumió el valor mínimo que se puede registrar en los subíndices, cuyo valor es de 29 que corresponde a valores de solidos disueltos totales mayores a $500 \mathrm{mg} / \mathrm{L}$.

Los indicadores de contaminación fecal más utilizados en los diferentes países son los Coliformes totales (CT) y los Coliformes fecales (CF). Sin embargo, numerosos estudios revelan que no existe relación significativa entre estos indicadores, sus cuantificaciones y las enfermedades relacionadas con el baño en agua de mar [14].

Algunos autores, [15], observaron que los Coliformes fecales y los Enterococos son los indicadores más apropiados para determinar la presencia de contaminación de origen fecal en el cuerpo de agua. Fujioka et al. [16], sostienen que E. coli y Enterococos son ubicuos y pueden persistir por largos periodos de tiempo en agua y suelos tropicales y subtropicales.

En el ecosistema marino los vibrios juegan funciones importantes como biodegradación de la materia orgánica y regeneración de nutrientes [17], además pueden actuar como patógenos de organismos acuáticos de importancia comercial, o bien afectar al hombre [18].

Es importante estudiar a los vibrios que actúan de forma deletérea sobre organismos de importancia comercial, debido a las consecuencias económicas que este produce y descubrir los factores que ocasionan este accionar es aún una tarea pendiente.

Por estas razones, hemos incluidos estos elementos como factores claves en la determinación de la vulnerabilidad de la población en la ciudad de Portobelo.

En el caso de la presencia de Vibrio fue determinado en la Bahía de Portobelo y se encontró en el agua de mar dos tipos: Vibrio Cholerae y Vibrio Parahaemolyticus.

Tabla 5. Valores Promedios para cada estación de muestreo obtenidos en el periodo de 2017 - 2019 en Portobelo.

\begin{tabular}{|c|c|c|c|c|c|c|c|c|c|}
\hline Parámetros & E1 & E2 & E3 & E4 & E5 & E6 & E7 & E8 & E9 \\
\hline $\mathrm{pH}$ & 7.6 & 7.6 & 7.6 & 7.6 & 7.6 & 7.5 & 7.6 & 7.5 & 7.6 \\
\hline $\begin{array}{c}\mathrm{PO}_{4} \\
(\mathrm{mg} / \mathrm{l})\end{array}$ & 0.0 & 0.1 & 0.1 & 0.1 & 0.1 & 0.1 & 0.1 & 0.0 & 0.0 \\
\hline
\end{tabular}


Ariel Antonio Grey G. | Alides Canto | Yarisel González | Kelsyn Barria | Itzel Castillo

\begin{tabular}{|c|c|c|c|c|c|c|c|c|c|}
\hline $\begin{array}{c}\text { S.T. } \\
(\mathrm{mg} / \mathrm{l})\end{array}$ & $\begin{array}{c}2.8 \\
3 \mathrm{E} \\
4\end{array}$ & $\begin{array}{c}3.2 \\
8 \mathrm{E} \\
4\end{array}$ & $\begin{array}{c}3.0 \\
0 \mathrm{E} \\
4\end{array}$ & $\begin{array}{c}2.98 \\
\text { E4 }\end{array}$ & $\begin{array}{c}2.6 \\
9 \mathrm{E} \\
4\end{array}$ & $\begin{array}{c}2.7 \\
1 \mathrm{E} \\
4\end{array}$ & $\begin{array}{c}2.9 \\
6 \mathrm{E} \\
4\end{array}$ & $\begin{array}{c}2.8 \\
7 \mathrm{E} 4\end{array}$ & $\begin{array}{c}2.6 \\
9 \mathrm{E} 4\end{array}$ \\
\hline $\begin{array}{c}\mathrm{NO}_{3} \\
(\mathrm{mg} / \mathrm{l})\end{array}$ & 1.3 & 1.5 & 1.3 & 1.2 & 1.4 & 1.2 & 0.9 & 1.1 & 0.9 \\
\hline $\begin{array}{c}\text { O.D. } \\
(\mathrm{mg} / \mathrm{l})\end{array}$ & 6.1 & 6.0 & 6.1 & 6.1 & 5.8 & 5.8 & 5.8 & 6.1 & 6.2 \\
\hline $\begin{array}{c}\text { Turb. } \\
\text { (UTM) }\end{array}$ & $\begin{array}{c}19 . \\
7 \\
\end{array}$ & $\begin{array}{c}31 . \\
8 \\
\end{array}$ & $\begin{array}{c}37 . \\
4 \\
\end{array}$ & 24.6 & $\begin{array}{c}25 . \\
2 \\
\end{array}$ & $\begin{array}{c}18 . \\
6 \\
\end{array}$ & $\begin{array}{c}34 . \\
7 \\
\end{array}$ & $\begin{array}{c}30 . \\
2 \\
\end{array}$ & $\begin{array}{c}24 . \\
7 \\
\end{array}$ \\
\hline $\begin{array}{c}\text { Temp. } \\
\left({ }^{\circ} \mathrm{C}\right)\end{array}$ & $\begin{array}{c}25 . \\
58 \\
\end{array}$ & $\begin{array}{l}25 . \\
75 \\
\end{array}$ & $\begin{array}{l}25 . \\
75 \\
\end{array}$ & $\begin{array}{c}25.7 \\
5 \\
\end{array}$ & $\begin{array}{l}25 . \\
75 \\
\end{array}$ & $\begin{array}{l}25 . \\
92 \\
\end{array}$ & $\begin{array}{l}25 . \\
92 \\
\end{array}$ & $\begin{array}{l}25 . \\
92 \\
\end{array}$ & $\begin{array}{l}25 . \\
92 \\
\end{array}$ \\
\hline $\begin{array}{l}\mathrm{DBO}_{5} \\
(\mathrm{mg} / \mathrm{l})\end{array}$ & 0.4 & 0.7 & 1.1 & 0.9 & 0.8 & 0.7 & 1.4 & 0.5 & 0.8 \\
\hline $\begin{array}{l}\text { C. T. } \\
\text { (UFC) }\end{array}$ & $\begin{array}{c}1.1 \\
5 \mathrm{E} \\
4\end{array}$ & $\begin{array}{c}2.0 \\
9 \mathrm{E} \\
4\end{array}$ & $\begin{array}{c}7.1 \\
4 \mathrm{E} \\
3\end{array}$ & $\begin{array}{c}4.02 \\
\text { E4 }\end{array}$ & $\begin{array}{c}5.1 \\
1 \mathrm{E} \\
4\end{array}$ & $\begin{array}{c}5.3 \\
4 \mathrm{E} \\
4\end{array}$ & $\begin{array}{c}3.8 \\
2 \mathrm{E} \\
4\end{array}$ & $\begin{array}{c}3.8 \\
8 \mathrm{E} 4\end{array}$ & $\begin{array}{c}3.4 \\
0 \mathrm{E} 4\end{array}$ \\
\hline
\end{tabular}

Luego de obtener el subíndice de cada parámetro, según la ecuación (1), estos valores se deben multiplicar por el peso relativo correspondiente a cada parámetro, para luego realizar la sumatoria y así obtener el Índice de Calidad de Agua. Los resultados se resumen la tabla 6.

Tabla 6. Subíndice de cada parámetro por estación de muestreo

\begin{tabular}{|c|c|c|c|c|c|c|c|c|c|}
\hline \multirow{2}{*}{ Parámetros } & \multicolumn{10}{|c|}{ Estaciones } \\
\cline { 2 - 11 } & E1 & E2 & E3 & E4 & E5 & E6 & E7 & E8 & E9 \\
\hline $\mathrm{pH}$ & 87.5 & 87.5 & 87.5 & 87.5 & 87.5 & 87.5 & 87.5 & 87.5 & 87.5 \\
\hline Fosfato & 98 & 95 & 95 & 96 & 96 & 96 & 97 & 99 & 99 \\
\hline Nitrato & 93.3 & 91.7 & 93.3 & 93.8 & 92.1 & 93.8 & 95.0 & 94.2 & 95.0 \\
\hline $\begin{array}{c}\text { Oxígeno } \\
\text { Disuelto }\end{array}$ & 61.3 & 60.0 & 61.3 & 61.3 & 56.3 & 56.3 & 56.3 & 61.3 & 62.5 \\
\hline DBO $_{5}$ & 96.5 & 92.2 & 90.0 & 90.9 & 92.2 & 92.2 & 85.2 & 95.2 & 92.2 \\
\hline Turbiedad & 62.5 & 50.8 & 45 & 57.5 & 56.7 & 64.2 & 47.5 & 51.7 & 57.5 \\
\hline $\begin{array}{c}\text { Coliformes } \\
\text { Totales }\end{array}$ & 50 & 45.2 & 53.6 & 39 & 37 & 36.4 & 40 & 40 & 40.9 \\
\hline Temperatura & 76.7 & 80.0 & 80.0 & 80.0 & 80.0 & 81.1 & 81.1 & 81.1 & 81.1 \\
\hline $\begin{array}{c}\text { Solidos } \\
\text { Disueltos }\end{array}$ & 29 & 29 & 29 & 29 & 29 & 29 & 29 & 29 & 29 \\
\hline
\end{tabular}

El promedio del índice de calidad de agua de todas las estaciones corresponde a $71.71 \mathrm{el}$ cual se encuentran en una calidad de agua buena que corresponde al intervalo entre $71-90$. La mejor condicione de calidad del agua se presentó a la estación uno (1) con un índice de 74 y la menor calidad se presenta en la estación siete (7) con un índice de 69 de calidad.

Tabla 7. Resultados del índice de calidad de agua para cada muestreo

\begin{tabular}{|c|c|c|c|c|c|c|c|c|c|}
\hline \multirow{2}{*}{ Parámetros } & \multicolumn{10}{|c|}{ Estaciones } \\
\cline { 2 - 11 } & E1 & E2 & E3 & E4 & E5 & E6 & E7 & E8 & E9 \\
\hline \multirow{2}{*}{$\mathrm{pH}$} & 11. & 11. & 11. & 11. & 11. & 11. & 11. & 11. & 11. \\
& 4 & 4 & 4 & 4 & 4 & 4 & 4 & 4 & 4 \\
\hline Fosfato & 7.8 & 7.6 & 7.6 & 7.7 & 7.7 & 7.7 & 7.8 & 7.9 & 7.9 \\
\hline Nitrato & 9.3 & 9.2 & 9.3 & 9.4 & 9.2 & 9.4 & 9.5 & 9.4 & 9.5 \\
\hline
\end{tabular}

\begin{tabular}{|c|c|c|c|c|c|c|c|c|c|}
\hline \multirow{2}{*}{ Oxígeno Disuelto } & 10. & 10. & 10. & 10. & 9.6 & 9.6 & 9.6 & 10. & 10. \\
& 4 & 2 & 4 & 4 & & & & 6 \\
\hline \multirow{2}{*}{$\mathrm{DBO}_{5}$} & 13. & 12. & 12. & 12. & 12. & 12. & 11. & 13. & 12. \\
& 5 & 9 & 6 & 7 & 9 & 9 & 9 & 3 & 9 \\
\hline Turbiedad & 6.3 & 5.1 & 4.5 & 5.8 & 5.7 & 6.4 & 4.8 & 5.2 & 5.8 \\
\hline $\begin{array}{c}\text { Coliformes } \\
\text { Totales }\end{array}$ & 5.5 & 5.0 & 5.9 & 4.3 & 4.1 & 4.0 & 4.4 & 4.4 & 4.5 \\
\hline Temperatura & 7.7 & 8.0 & 8.0 & 8.0 & 8.0 & 8.1 & 8.1 & 8.1 & 8.1 \\
\hline Solidos Disueltos & 2.0 & 2.0 & 2.0 & 2.0 & 2.0 & 2.0 & 2.0 & 2.0 & 2.0 \\
\hline$\Sigma$ & 74 & 71 & 72 & 72 & 71 & 71 & 69 & 72 & 73 \\
\hline
\end{tabular}

Para conocer un poco sobre las amenazas y vulnerabilidad que enfrenta la comunidad se realizaron encuestas a los pobladores de la ciudad de Portobelo, dicha encuesta fue realizada en marzo 2019 a un total de 74 viviendas.

Para las amenazas socio naturales, las encuestas revelaron que el $69 \%$ de la población ha sufrido de inundaciones y deslizamientos de tierra, lo que se clasifica como un grado de amenaza alta con un puntaje de 4; para las amenazas antropogénicas se analizó el ICA, en donde se obtuvo como resultado 71.71 lo que indica una calidad de agua buena a regular y se clasifica como un grado de amenaza de moderado a bajo.

Para definir la amenaza total presente en la comunidad de Portobelo se calculó el promedio de los indicadores evaluados, en donde se obtiene que la amenaza total es de 2.

Para las vulnerabilidades físicas los indicadores que se evaluaron fueron: el tipo de material de las viviendas y la densidad de población de la zona de estudio. Las encuestas revelaron que el $100 \%$ de la población encuestadas habitan en viviendas adecuadas, lo que indica un grado de vulnerabilidad bajo con un puntaje de 1. Con respecto a la densidad se obtuvo que el número de personas por viviendas es de aproximadamente 4 y para un total de 74 viviendas encuestadas da como resultado una densidad de 181 personas, lo que entre en un grado de vulnerabilidad moderado con un puntaje de 3 .

Para las vulnerabilidades socioeconómicas se obtuvo que el $50 \%$ de los habitantes están desempleados lo que genera un grado de vulnerabilidad moderado. Otro indicador evaluado es el rango de salario en donde el $46 \%$ de la población encuestada recibe un salario menor de $500.00 \$$, lo que indica un grado de vulnerabilidad moderado. También se evaluó la población que no cuenta con casa propia, que en total es un $12 \%$ del total, lo que indica un grado de vulnerabilidad bajo. Toda comunidad debe brindar los servicios básicos necesarios a sus 
habitantes como lo es el sistema de acueducto, según la encuesta realizada el $100 \%$ de la población no cuenta con el servicio de agua potable, lo que da como resultado un grado de vulnerabilidad muy alta. Otros de los servicios básicos que debe tener una comunidad es Energía eléctrica, todas las viviendas encuestadas cuentan con energía eléctrica lo que se clasifica como un grado de vulnerabilidad muy bajo. También se evaluó si la población cuenta con servicios de internet, radio y teléfono, en donde ambos se encuentran en un grado de vulnerabilidad bajo.

Para las vulnerabilidades ambientales se evaluaron indicadores que permiten saber si la comunidad de Portobelo genera impactos negativos en el ambiente.

Uno de estos indicadores estudiados fue la el contar con un sistema de alcantarillado, en donde el $40 \%$ de las viviendas encuestadas cuentan con una red de desagüe lo que dan un grado de vulnerabilidad moderado. Otro indicador es si cuenta con un tratamiento de aguas residuales ya que es un factor importante para reducir los impactos al ecosistema, sin embargo, Portobelo no cuenta con una planta de tratamiento de aguas residuales lo que es un grado de vulnerabilidad muy alto. Otro factor de influencia en el ambiente es la frecuencia con que se eliminan los desechos sólidos, en donde el 13\% de la población encuestada indico que la recolección se realiza una vez por semana, esto genera un grado de vulnerabilidad bajo. Sin embargo, no concuerda con lo observado, la gran perturbación con presencia de desechos en el mar, quebradas, zonas de manglares y zanjas.

La vulnerabilidad educativa, indica si la población esta adecuadamente preparada para cualquier tipo de amenazas, para esto se evaluó indicador como si la población cuenta con estudios académico, en donde el $19 \%$ de los encuestados no poseen estudios lo que indica un grado de vulnerabilidad bajo. Sin embargo, el 58\% llegaron hasta primaria o secundaria. Lo que hace a la población más vulnerable. Otro factor de importancia es si la ciudadanía conoce sobre las medidas preventivas ante amenazas como inundaciones y deslizamiento de tierra y que estos son fenómenos que se agravan debido a las actividades humanas, en los últimos años el cambio climático tiene gran influencia sobre estos fenómenos lo que provoca que las comunidades se vean cada vez más afectadas; según las encuestas realizadas el $81 \%$ de las personas no conocen medidas preventivas antes inundaciones y deslizamientos lo que genera un grado de vulnerabilidad alto, con respecto al conocimiento del cambio climático el $58 \%$ de los encuestados no conocen sobre el tema, lo que genera un grado de vulnerabilidad moderado.

Adicional a estos elementos analizados, otro factor de impacto, las viviendas se encuentran muy cerca del manglar lo que se considera un grado de amenaza muy alto.

Presenta un grado de amenaza alto ya que el $84 \%$ de la población utiliza el agua para beber, y como ya mencionamos anteriormente, Portobelo no cuenta con agua potable, lo que se considera una ponderación de 4.

\begin{tabular}{|c|c|c|c|}
\hline Vulnerabilidad & Indicador & Resultado & Puntaje \\
\hline \multirow{2}{*}{ Física } & $\begin{array}{l}\text { Porcentaje de las viviendas } \\
\text { con material inadecuado }\end{array}$ & $0 \%$ & 1 \\
\hline & $\begin{array}{l}\text { Habitantes en Ciudad de } \\
\text { Portobelo área de estudio }\end{array}$ & 181 & 3 \\
\hline \multirow{8}{*}{ Socioeconómica } & $\begin{array}{l}\text { Porcentaje de la población } \\
\text { desempleada }\end{array}$ & $52 \%$ & 3 \\
\hline & $\begin{array}{l}\text { Porcentaje de la población } \\
\text { que gana menos de } 500.00 \$\end{array}$ & $46 \%$ & 3 \\
\hline & $\begin{array}{l}\text { Porcentaje de población sin } \\
\text { casa propia }\end{array}$ & $12 \%$ & 1 \\
\hline & $\begin{array}{l}\text { Porcentaje de viviendas que } \\
\text { no cuentan con sistemas de } \\
\text { acueducto }\end{array}$ & $100 \%$ & 5 \\
\hline & $\begin{array}{l}\text { Porcentaje de viviendas que } \\
\text { no cuentan con energía } \\
\text { eléctrica }\end{array}$ & $0 \%$ & 1 \\
\hline & $\begin{array}{l}\text { Porcentaje de viviendas sin } \\
\text { Internet }\end{array}$ & $64 \%$ & 3 \\
\hline & $\begin{array}{l}\text { Porcentaje de viviendas sin } \\
\text { teléfonos }\end{array}$ & $64 \%$ & 3 \\
\hline & $\begin{array}{l}\text { Porcentaje de viviendas sin } \\
\text { radio }\end{array}$ & $25 \%$ & 2 \\
\hline \multirow{3}{*}{ Ambiental } & $\begin{array}{l}\text { Porcentaje de viviendas sin } \\
\text { sistema de alcantarillado }\end{array}$ & $100 \%$ & 5 \\
\hline & $\begin{array}{l}\text { No cuenta con sistema de } \\
\text { tratamiento de aguas } \\
\text { residuales }\end{array}$ & $100 \%$ & 5 \\
\hline & $\begin{array}{l}\text { Porcentaje de viviendas sin } \\
\text { sistemas de recolección de } \\
\text { los desechos }\end{array}$ & $13 \%$ & 1 \\
\hline \multirow{3}{*}{ Educativa } & $\begin{array}{l}\text { Porcentaje de la población } \\
\text { que no poseen estudios }\end{array}$ & $19 \%$ & 2 \\
\hline & $\begin{array}{l}\text { Porcentaje de población que } \\
\text { no conocen medidas } \\
\text { preventivas }\end{array}$ & $81 \%$ & 4 \\
\hline & $\begin{array}{l}\text { Porcentaje que no conocen } \\
\text { sobre el cambio climático }\end{array}$ & $58 \%$ & 3 \\
\hline
\end{tabular}


Con los indicadores ya evaluados para los distintos tipos de vulnerabilidades se puede obtener que las vulnerabilidades física en la ciudad de Portobelo es de 2.0 lo que se clasifica como un grado de vulnerabilidad moderado a bajo, las vulnerabilidades socioeconómicas es de 3.6 con un grado de vulnerabilidad moderada a alta, para las vulnerabilidades ambientales da como resultado 3.7 con un grado de vulnerabilidad moderado a alto, y con respecto a las vulnerabilidades educativas da como resultado 2.7 lo que es un grado de vulnerabilidad bajo moderado.

Con los resultados obtenidos en los cálculos de amenaza y vulnerabilidad en la ciudad de Portobelo, y aplicando la ecuación (4) se obtiene que el IRV es de 3.0, lo que se clasifica en un grado moderado, a pesar de que presento grados de vulnerabilidades muy alto estos factores no tienen un impacto sobre el resultado final, pero aun así se debe prestar atención a los factores en donde se presentaron puntajes muy alto de alerta. Tales factores los podemos resumir, la contaminación de los manglares por desechos, las descargas de aguas residuales directo al mar, la ausencia de un sistema de potabilización del agua y el turismo informal no sostenible. Se debe implementar medidas de mitigación y concientización de la población para evitar que las amenazas sea de un impacto mayor provocando desastres.

\section{Conclusiones y recomendaciones}

El Objetivo de la investigación era lograr determinar un índice de riesgo y vulnerabilidad en función al análisis de la calidad de las aguas superficiales marino-costeras a partir de evaluaciones de factores socioeconómicos en Portobelo, provincia de Colon. Se logra comprobar que, a través de estos factores, se pudo determinar el índice de riesgo y vulnerabilidad, aplicado a poblaciones costeras.

El aporte principal de este trabajo consiste en el diseño de una metodología clara y fácil de usar para poder determinar de un Índice de Riesgo y vulnerabilidad a partir de análisis de la calidad de agua y factores socioeconómicos.

La comunidad de Portobelo presenta una amenaza alta para Inundaciones, deslizamiento de tierra y contaminación del agua en la zona costera de la ciudad.

En cuanto a las vulnerabilidades se presentó un grado de vulnerabilidad moderada a alta, un resultado alarmante, donde las vulnerabilidades socioeconómicas y ambientales presentaron resultados preocupantes debido a su alto potencial turística y alto valor histórico, por lo que se considera que se le deben prestar suma atención a diversas condiciones; no cuenta con una planta de tratamiento de aguas residuales, lo que afecta a la zona costera debido a que todos las aguas residuales son descargas directamente al mar, afectando al ecosistema marino y de una manera indirecta al ser humano.

Fundamental, es la construcción de una planta de tratamiento de aguas residuales, la conexión de las casas a un sistema de alcantarillado y la urgente necesidad de un sistema de potabilización del agua para consumo humano. Un manejo eficiente de los desechos sólidos, para evitar la presión ambiental sobre los manglares adyacente a la zona costera. En cuanto a la calidad del agua, esta presenta una alta condición, lo cual debería existir una prohibición para el uso de la zona costera, para actividades de contacto directo, pesca y consumo de especies de la zona, por los altos niveles de Coliformes, enterococos y vibrio presente en el cuerpo de agua.

Se recomienda seguir haciendo este tipo de estudios en las diversas zonas costeras del país, para poder así presentar datos de riesgos que ayuden a las comunidades a disminuir sus vulnerabilidades y así poder prepararse ante cualquier tipo de amenazas.

\section{AGRADECIMIENTOS}

Los autores agradecen al Servicio Aéreo Naval de Panamá, Autoridad Portuaria de Panamá, al Laboratorio de Ingeniería Sanitaria de la Facultad de Ingeniería Civil de la Universidad Tecnológica de Panamá, por su valiosa colaboración y participación en las diversas campañas de muestreos y análisis de muestras de algunos parámetros ambientales y a la Secretaria Nacional de Ciencia, Tecnología e Innovación (SENACYT) por el financiamiento para realizar esta investigación, a través del Proyecto FID-16-011.

\section{REFERENCIAS}

[1] INVEMAR, 2014. Vulnerabilidad de la población costera frente a la contaminación orgánica y microbiológica en la bahía de buenaventura.

[2] Grey, A. (2007). "Estudio Comparativo de la Calidad de las Aguas Costera Durante el Periodo Seco y Lluvioso en la Bahía de Manzanillo de la Ciudad de Colón”. Tesis de Maestría.

[3] D’Croz, L., Martines, V. y Arosemena, G. (1994) "Inventario Biológico del Canal de Panamá. El Estudio 
Marino". SCIENTIA. Revista de Investigación de la Universidad de Panamá.

[4] Alonso, David; Sierra, Paula; Fontalvo, Martha \& Arias, Francisco. (2003). “Conceptos y Guía Metodológica para el Manejo Integrado de Zonas Costeras en Colombia, manual 1: Preparación, caracterización y diagnóstico". INVEMAR. Serie documentos Generales No. 12. 94 p. Santa Marta.

[5] Garay, J. y A. Vélez. (2004). "Programa Nacional de Investigación, Evaluación, Prevención, Reducción y Control de Fuentes Terrestres y Marinas de Contaminación al Mar-PNICM". Santa Marta: Instituto de Investigaciones Marinas y Costeras "José Benito Vives de Andréis"INVEMAR. Santa Marta.134 p.

[6] Tejada, C., Castro, L., Navarrete, A., Cardona, T., Otero, L., Afanador, F., y otros (2003). "Panorama de la Contaminación Marina del Pacífico Colombiano". San Andrés de Tumaco: Centro Control Contaminación del Pacífico Colombiano. Ed. DIMAR. Serie de Publicaciones Espaciales Vol., 3. 120 p. San Andrés de Tumaco.

[7] Rojas, X., P. Sierra-Correa., P. Lozano-Rivera y A. López. (2010). "Guía metodológica para el manejo integrado de las zonas costeras en Colombia, Manual 2: planificación de la zona costera". Serie de Documentos Generales INVEMAR No.44, Santa Marta. 74p.

[8] M. Castro, J. Almaida, J. Ferrer y D. Díaz (2014). "Indicadores de la calidad del agua: evolución y tendencias a nivel global". Ingeniería Solidaria, vol. 10, n. ${ }^{\circ}$ 17, pp. 111124.

[9] Samboni Ruiz, N. E., Carvajal Escobar, Y., \& Escobar, J. C. (2007). "Revisión de parámetros físicoquímicos como indicadores de calidad y contaminación del agua". Ingeniería e investigación, Universidad Nacional de Colombia Bogotá, Colombia, vol. 27, núm. 3, pp. 172-181.
[10] Ott, W. (1978). "Environmental Indices., Theory and Practice, Aa Science, Ann Arbor, Michigan”.

[11] V. Guillén, H. Teck, B. Kohlmann y J. Yeomans (2012). "Microorganismos como bioindicadores de la Calidad del Agua, Tierra tropical: sostenibilidad, ambiente y sociedad", vol. 8, n. $^{\circ} 1$, pp. $65-93$

[12] M. Wills y K. N. Irvine (1996). "Application of the National Sanitation Foundation Water Quality Index in Cazenovia Creek, NY, Pilot Watershed Management Project”. Middle States Geographer, pp. 95-10.

[13] Wilches Chaux, G. (1998). "Auge, caída y levantada de Felipe Pinillo, mecánico y soldador o yo voy a correr el riesgo" (No. 658.155 W5).

[14] U.S.EPA (United States Environmental Protection Agency) (1986). Quality Criteria for Water EPA 440/5-86-001. 1 May 1986. Office of Water Regulations and Standards, Washington D.C., USA.

[15] Herrera A, Suárez P. Indicadores bacterianos como herramientas para medir la calidad ambiental del agua costera. INCI. 2005; 30(3):171- 176

[16] Fujioka, R.; C. Sian-Denton; M. Borja; J. Castro and K. Morphew (1999). Soil: the environmental source of Escherichia coli and enterococci in Guam's streams. J. Appl. Microbiol. Symp. Suppl. 85: 83S- 89S.

[17] Cavallo, R.A., \& Stabili, L. (2002). Presence of vibrios in seawater and Mytilus galloprovincialis (Lam.) from the Mar Piccolo of Taranto (Ionian Sea). Water Research, 36, 37193726 .

[16] Thompson JR, MA Randa, LA Marcelino, A Tomita-Mitchell, E Lim \& MF Polz. 2004. Diversity and dynamics of a North Atlantic coastal Vibrio community. Applied and Environmental Microbiology 70: 4103-4110. 